\title{
SABER DEL MAESTRO Y APORTE DE LAS TIC EN LA FORMACIÓN DEL CAPITAL HUMANO EN LA ESCUELA
}

\author{
TEACHER'S KNOWLEDGE AND \\ CONTRIBUTION OF TIC IN THE \\ FORMATION OF HUMAN CAPITAL \\ IN SCHOOL
}

\section{Diana Clemencia Sánchez Giraldo ${ }^{1}$}

Maria Janeth Cometa Valencia ${ }^{2}$

\section{RESUMEN}

La enseñanza de hoy y de las nuevas generaciones está siendo influenciada e intervenida por las Nuevas Tecnologías de la Información y la Comunicación, las cuales, cada vez insertan nuevos retos, posibilidades y oportunidades en el ámbito de la educación, por lo que, corresponde al docente y particularmente el que se desenvuelve en contextos rurales del municipio colombiano, adelantar un proceso de apropiación y gestión del conocimiento respecto del uso y manejo de estas tecnologías, así como 1 Magister en Educación. Candidata a doctora en Educación de la Universidad de Caldas. Docente investigadora Universidad Católica de Manizales. Maestría en Gestión del conocimiento educativo. Email. disanchez@ ucm.edu.co https://orcid.org/0000-0003-4339-2318 2

Magister en Gestión del conocimiento educativo. Docente Secretaria de educación y cultura del Cauca- Centro Educativo La Chicueña, Municipio del Tambo Cauca. Email: mariyeanneth@gmail.com. https://orcid.org/0000-00018796-3755 del saber y de la gestión del talento humano que se educa en la escuela.

Lo anterior, demanda un docente con nuevas habilidades y un proceso continuo de formación en las áreas como el uso y apropiación de las TIC, que permitan a toda la comunidad educativa, el logro de una transformación del conocimiento y con él, de las condiciones de vida del contexto de la escuela. Por ello, esta investigación analizó la contribución y aporte que hacen los docentes a través de sus saberes en el uso de las TIC, al desarrollo del capital humano en el Centro Educativo La Chicueña, Municipio de El Tambo Cauca, identificando los tipos de saberes que poseen los docentes en relación con el manejo y uso de las TIC, así como se caracterizó a partir del uso de ellas, la gestión del proceso de desarrollo del capital humano en el Centro Educativo y por último se relacionaron los saberes del docente 
y la innovación TIC en las prácticas pedagógicas desarrolladas en la escuela.

Palabras Clave: Comunidades de Práctica, Gestión del Conocimiento, Práctica Pedagógica e Innovadora, Aprendizaje Organizacional.

\section{INTRODUCCIÓN}

En el contexto globalizado del uso de las Tecnologías de la Información y la Comunicación (TIC), estas se han convertido en una herramienta que facilita en cierta medida la vida de los seres humanos, considerando su adecuado uso en diferentes tareas de la cotidianidad humana, siendo la formación y la educación, uno de los aspectos donde más incidencia tienen, por cuanto, determinan nuevas maneras y tendencias en la formación, a partir de una innovadora metodología, pedagogía y didáctica, así como, mejores formas de compartir la información que el conocimiento brinda a un conglomerado social.

En esta globalización, es inevitable no analizar la relación existente entre la gestión del conocimiento y el uso de las herramientas TIC, por cuanto, estas últimas aportan una rápida y adecuada generación, trasmisión y difusión del conocimiento, ya sea en un proceso formal (El educativo en la escuela) o informal (En casa, la comunidad o en la sociedad), ya sea a través de modelos pedagógicos o de la información suministrada a través de distintas formas, herramientas o estrategias de comunicación.

Sin embargo, en al ámbito educativo de la escuela rural, es escasa la atención que la dinámica institucional le presta a la gestión de las TIC y las formas de cómo estos son trasmitidos a la población en formación; desatención que radica en el hecho de la escaza sistematización tecnológica del proceso educativo en la Institución Educativa y de la incipiente gestión de los saberes del docente frente al manejo de estas, lo que, disminuye el desarrollo de los procesos tanto administrativos, operativos y los pedagógicos, además que mengua el espacio para la reflexión sobre su propio hacer, generando que en las aulas escolares de una gran parte de las escuelas rurales, se siga enseñando de forma anacrónica a los estudiantes y que el proceso enseñanza y aprendizaje no avance hacia la construcción de espacios significativos.

Lo anterior, se profundiza considerando los avances tecnológicos que se viven continuamente en el mundo actual, en la que la sociedad y en ella, particularmente los niños son consumidores dosificados, aprendiendo unos de otros, o a través de las redes sociales o plataformas virtuales, lo que obliga a crear en el proceso educativo, entornos colectivos y colaborativos de aprendizaje, en donde el conocimiento individual se transforme en conocimiento organizacional y en donde exista transferencia de conocimientos que generen desarrollo de competencias relacionadas a la gestión del conocimiento y produzca un impacto social y el empoderamiento de los estudiantes, para que estos, contribuyan a la resolución de problemas.

De continuar con el actual modelo pasivo de aprendizaje, en el que el docente es quién habla y dirige, en tanto que, los estudiantes escuchan, escriben sin ser proactivo y sin creatividad, la educación seguirá siendo monótona y poco emotiva, respecto de las transformaciones que vive el mundo actual. No obstante, el acelerado desarrollo las TIC y fundamentalmente en el proceso educativo, en países tercermundistas como Colombia y en regiones como el Cauca, este desarrollo no ha sido el óptimo por cuanto, no existen las condiciones mínimas de dotación de equipos asociados a estas tecnologías, así como, la incipiente cobertura de conectividad en zonas rurales, a donde no llega señal de internet y servicios conexos, impiden dicha conectividad y acceso a plataformas que facilitan el acceso 
a la gestión del conocimiento que, a través de ellas, se ofrece.

Sin embargo, según Velasco (2014), en Colombia, a partir de la creación del Sistema Nacional de Ciencia, Tecnología e Innovación, los últimos gobiernos, han establecido dentro de los Planes de Desarrollo Nacional, programas y proyectos encaminados al fortalecimiento de las TIC en todo el territorio, buscando atemperar a la modernidad de estas tecnologías a todo el país.

A través de estos programas e iniciativas, se ha buscado incidir en el proceso educativo de los colombianos, utilizando herramientas tecnológicas (Conectividad a internet, computadores, video beam, celulares inteligentes, plataformas virtuales) para acceder a la información en diferentes áreas del conocimiento y facilitar al docente, formas pedagógicas y desarrollos metodológicos para proporcionar dicho conocimiento que fortalecen el proceso educativo en la escuela convencional. En virtud de esto, surge la pregunta de investigación: ¿Cuál es la contribución que hacen los docentes a través de sus saberes en lo relacionado con el uso de las TIC, y su aporte al desarrollo del capital humano en el Centro Educativo la Chicueña, Municipio de El Tambo Cauca?

\section{METODOLOGÍA}

Para el desarrollo de esta investigación se recurrió al enfoque de Investigación Cualitativa de corte Descriptivo, buscando analizar las características de la formación del talento humano del Centro Docente y la incidencia de las TIC en la formación del capital humano, obteniendo a partir de la observación, datos e información, así como la experiencia docente en el aula de clase, que fueron analizados y explicados en relación con el valor agregado que el uso de las TIC brinda en la formación estudiantil y en el capital humano docente y como esto incide en la población escolar y en la comunidad de la zona objeto de estudio.

Para ello, se inició con la recolección de datos, mediante la observación empírica o mediciones de algunas clases lobservación, entrevista, documentos escritos) construyendo, a partir de las relaciones descubiertas, sus categorías y proposiciones teóricas, pretendiendo descubrir una teoría que justifique esos datos, para luego, mediante el estudio y análisis de los fenómenos semejantes y diferentes, desarrollar una teoría explicativa. Seguidamente y citando a Walquer (1983), a partir de los datos, que se ordenan y clasifican, se generaron constructos y categorías, buscando la transferibilidad. Para terminar en la fase de Construcción, orientándose hacia el descubrimiento de los constructos analíticos o categorías que pudiesen obtenerse a partir del continuo comportamental; en donde el proceso de abstracción se revelan las unidades de análisis en el transcurso de la observación y descripción. (p. 10)

\section{FASES DE LA INVESTIGACIÓN}

3.1 Socialización y presentación de la propuesta de investigación ante la Institución Educativa

Consistió en la presentación del propósito de la investigación, así como la explicación de las etapas para adelantar la investigación. De igual manera, fue necesario desarrollar el estado del arte para ubicar el tema en el contexto internacional, nacional y local, estableciendo una matriz de objetivos y las categorías de análisis de acuerdo a cada uno de ellos.

3.2 Fase Dos. Identificación de técnicas y diseño de instrumentos para la recolección de la información

En esta fase se dispuso el diseño y estructuración de los instrumentos de recolección, así como de análisis de la información, tales como: Matriz de caracterización temática del saber docente; Encuestas sobre saberes del docente 
frente a las TIC; Diarios de campo y Plan de aula. De igual manera, se realizó la recolección de la información, para lo cual, se aplicaron los instrumentos según la caracterización previamente realizada y la contribución que cada docente brindó en dicho proceso.

3.3 Fase Tres. Procesamiento y análisis de la información

En esta fase se procedió a procesar la información obtenida y paralelo a ellos, se recurrió a la técnica de microanálisis de datos propuesta por Strauss y Corbin (2002) a partir de relatos registrados durante la observación y entrevista. Así mismo, se analizó la información procesada, orientada a documentar y contrastar información, lo que permitió cotejar los resultados obtenidos en las categorías definitivas del proceso, con la posición de la investigadora, los hallazgos obtenidos y el marco teórico referido.

\section{RESULTADOS}

A partir de la triangulación de las categorías identificadas del Saber Pedagógico, Manejo y Uso de las TIC y la Incidencia en el Capital Humano de la Institución Educativa La Chicueña del Municipio de El Tambo, la investigación mostró los siguientes resultados:

\subsection{Con Relación al Saber Pedagógico}

Díaz (2006) definió el saber pedagógico como los conocimientos construidos de manera formal e informal por el docente, entrañando sus valores, ideologías, actitudes, prácticas, todas sus creaciones, en un contexto histórico cultural, lo que emerge de interacciones personales e institucionales, que evolucionan, reestructuran, reconocen y permanecen en su vida. (p. 113).

Así mismo, Sánchez y González (2016) concibieron este saber cómo una forma de entender e interpretar el quehacer del profesor, que retoma su mirada de las concepciones de vida y cultura en el contexto histórico social en el que labora, a partir de la reflexión individual que realiza sobre las formas como piensa y representa su acción pedagógica. (p. 18)

Considerando lo anterior, el maestro como sujeto depositario del saber pedagógico, acopia la información preliminar y crea el conocimiento, convirtiéndolo luego en discurso y diseñando la didáctica, para posteriormente trasmitirla al alumno, utilizando la interdisciplinariedad, los elementos, los factores y ayudas que se configuren en la escena donde se desarrolla el proceso de enseñanza-aprendizaje, por lo que, esta investigación determinó respecto al saber pedagógico en referencia al uso y manejo de las TIC, que los docentes de la Institución Educativa se subcategorizan así:

- $\quad$ Con relación al Saber Formal, un 50\% obtuvieron el conocimiento sobre el manejo y uso de las TIC de manera formal, gracias a la puesta en marcha del Programa Computadores para Educar y en menor grado, porque adelantaron un programa de formación en esta área.

- $\quad$ Respecto del Saber informal, un $70 \%$ lo obtuvo de mediante la realización de algunos cursos, buscando apropiar conocimiento al respecto del uso y manejo de las TIC.

- $\quad$ Con relación al Saber Interactivo, un $80 \%$ adquirió el manejo pedagógico de las TIC, gracias a la interacción con otros docentes o personas.

- $\quad$ Y respecto del Saber Reflexivo, un $90 \%$ adquirió el conocimiento y manejo de las TIC, a través de la práctica de aprendizaje empírica, es decir, por si mismos y en el aula de clase. 
Tabla 1. Saber pedagógico de los docentes del Centro Educativo La Chicueña

\begin{tabular}{|c||c|c|c|c|c|}
\hline Sub & \multicolumn{1}{|c|}{ Pregunta } & $\begin{array}{c}\text { Totalmente } \\
\text { en } \\
\text { Desacuerdo } \\
\%\end{array}$ & $\begin{array}{c}\text { En } \\
\text { Desacuerdo } \\
\%\end{array}$ & $\begin{array}{c}\text { De Acuerdo } \\
\%\end{array}$ & $\begin{array}{c}\text { Totalmente } \\
\text { de Acuerdo } \\
\%\end{array}$ \\
\hline Formal & $\begin{array}{l}\text { Considero que el saber que } \\
\text { poseo en el manejo de las TIC } \\
\text { lo obtuve de manera formal. }\end{array}$ & 0 & 20 & 50 & 30 \\
\hline Informal & $\begin{array}{l}\text { Considero que parte de los } \\
\text { saberes sobre el manejo de } \\
\text { las TIC los obtuve de manera } \\
\text { informal. }\end{array}$ & 20 & 0 & 70 & 10 \\
\hline $\begin{array}{l}\text { Parte de los saberes que he } \\
\text { obtenido frente al manejo } \\
\text { de las TIC, son fruto de } \\
\text { mi interacción con los } \\
\text { estudiantes, docentes de } \\
\text { la institución, familiares y } \\
\text { amigos. }\end{array}$ & $\begin{array}{l}\text { El saber frente al manejo de } \\
\text { las TIC también lo he obtenido } \\
\text { de un proceso de reflexión } \\
\text { sobre la práctica pedagógica } \\
\text { que desarrollo día a día. }\end{array}$ & 0 & 0 & 80 & 10 \\
\hline Reflexivo
\end{tabular}

Fuente: Docente Investigadora. 2019

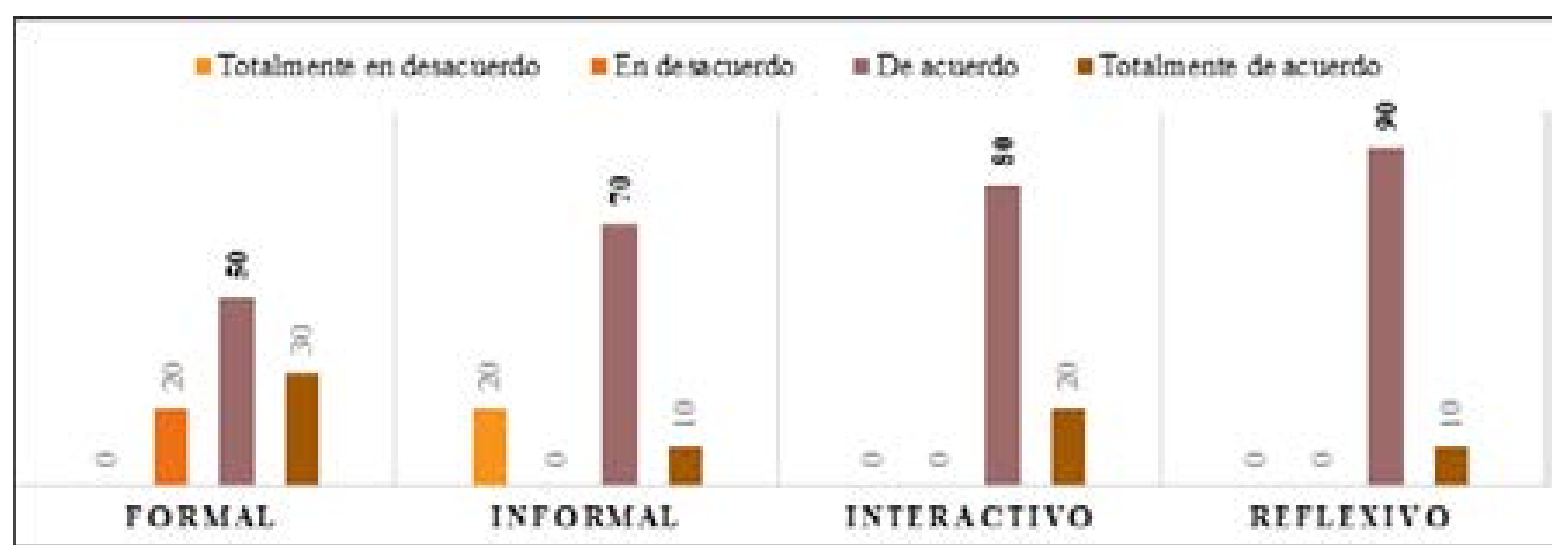

Gráfico 1. Porcentaje de obtención formal o informal del saber pedagógico de los docentes Fuente: Docente Investigadora. 2019 
De lo anterior, se analiza que el saber pedagógico respecto del manejo y uso de las TIC en el Centro Docente La Chicueña del Municipio de El Tambo Cauca, se obtiene mayoritariamente a partir del ejercicio reflexivo y la interacción del docente con sus pares u otras personas, así como en la necesidad de innovar en el desarrollo de la clase, más no obedeciendo a parámetros formales de adquisición de conocimientos sobre dicho uso y manejo.

\subsubsection{Saber contextual}

Referido este saber, como aquel que obtiene el docente gracias a la interacción y desde las tradiciones, creencias y cultura que posee el entorno donde enseña, forma y educa y de donde toma elementos y fundamentos para el mejor desarrollo del proceso educativo, buscando integrarlos al mejoramiento de la sociedad a la que sirve, en la investigación se obtuvo:

Tabla 2. Saber contextual de los docentes del Centro Educativo La Chicueña.

\begin{tabular}{|c|c|c|c|c|c|c|}
\hline Subcategoría & Pregunta & $\begin{array}{c}\text { Totalmente } \\
\text { en } \\
\text { Desacuerdo } \\
\%\end{array}$ & $\begin{array}{c}\text { En } \\
\text { Desacuerdo } \\
\%\end{array}$ & $\begin{array}{c}\text { De Acuerdo } \\
\%\end{array}$ & $\begin{array}{c}\text { Totalmente } \\
\text { de Acuerdo } \\
\%\end{array}$ & Total \% \\
\hline Tradiciones & $\begin{array}{l}\text { Los saberes } \\
\text { adquiridos en } \\
\text { el manejo de } \\
\text { las TIC también } \\
\text { se derivan de } \\
\text { las tradiciones } \\
\text { que aún se } \\
\text { conservan en } \\
\text { el contexto } \\
\text { familiar, escolar } \\
\text { y social. }\end{array}$ & 10 & 30 & 30 & 30 & 100 \\
\hline Creencias & $\begin{array}{l}\text { El saber en } \\
\text { el manejo de } \\
\text { las TIC está } \\
\text { asociado } \\
\text { también a mis } \\
\text { creencias. }\end{array}$ & 10 & 60 & 0 & 30 & 100 \\
\hline Cultura & $\begin{array}{l}\text { El saber frente } \\
\text { al manejo de las } \\
\text { TIC se encuentra } \\
\text { relacionado } \\
\text { al entorno } \\
\text { cultural al que } \\
\text { pertenezco. }\end{array}$ & 0 & 0 & 80 & 20 & 100 \\
\hline
\end{tabular}

Fuente: Docente Investigadora. 2019 
- $\quad$ Con relación a este saber, derivado de las tradiciones, el $30 \%$ de los docentes del Centro Educativo manifestaron que estas provienen del entorno familiar, escolar y social que comparten. Sin embargo, un $30 \%$ manifiestan que no, asociado esto a la no disponibilidad o acceso a la tecnología.

- $\quad$ En referencia a las creencias o interés de saber y conocer el manejo y uso de las TIC en el contexto educativo, un $30 \%$ considera que están asociadas a las creencias del docente, en tanto que un $60 \%$, consideran que obedece a factores que pasan por el quehacer profesional al querer profesional.

- Considerando la globalización e importancia de las TIC, un $80 \%$ de los docentes, plantea que dicho saber se relaciona con el entorno cultural en el que vive, que "lo obliga" a estar vigente en dicho saber tecnológico, para aportar en el proceso formativo y estar a la vanguardia de los procesos sociales, económicos, políticos, culturales y de innovación que vive el mundo actual.

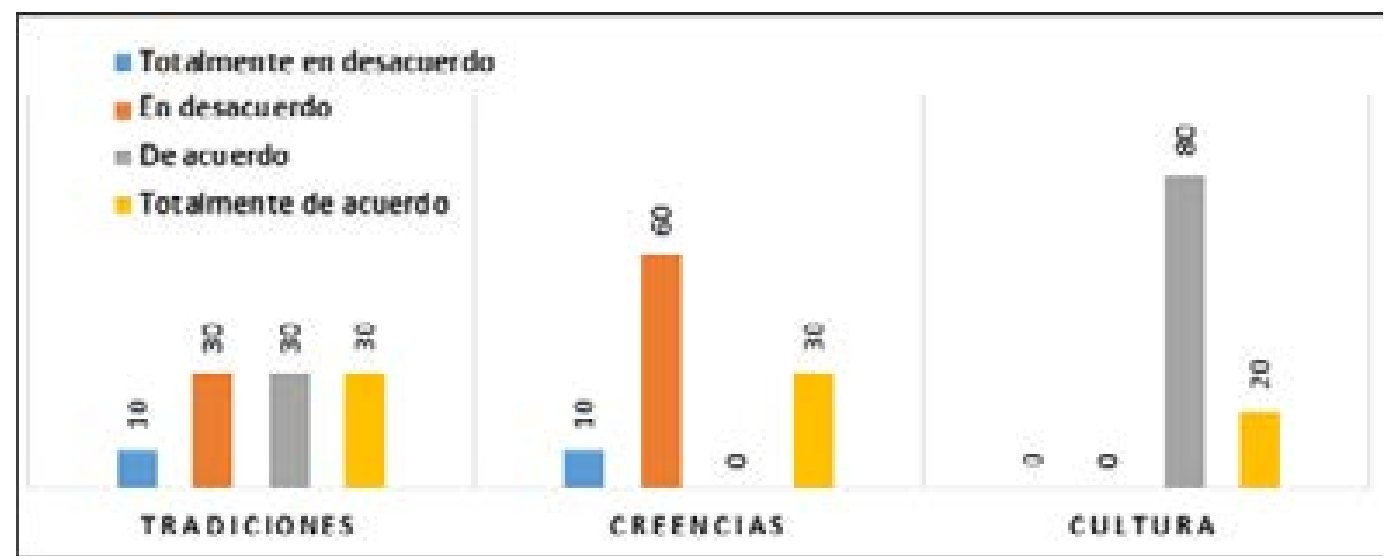

Gráfico 2. Porcentaje de apropiación del saber conceptual, respecto de las TIC Fuente: Docente Investigadora. 2019

\subsubsection{Saber especializado}

Considerando este saber del docente con base en la sistematización de experiencias, a partir de abordajes teóricos o la combinación de estos dentro de la cotidianidad de la formación, obteniendo los siguientes resultados: 
Tabla 3. Saber especializado de los docentes del Centro Educativo La Chicueña.

\begin{tabular}{|c|c|c|c|c|c|c|}
\hline Subcategoría & Pregunta & $\begin{array}{c}\text { Totalmente } \\
\text { en } \\
\text { Desacuerdo } \\
\%\end{array}$ & $\begin{array}{c}\text { En } \\
\text { Desacuerdo } \\
\%\end{array}$ & $\begin{array}{c}\text { De } \\
\text { Acuerdo } \\
\%\end{array}$ & $\begin{array}{c}\text { Totalmente } \\
\text { de Acuerdo } \\
\%\end{array}$ & Total \\
\hline Experiencias & $\begin{array}{l}\text { El saber en manejo } \\
\text { de TIC es el resultado } \\
\text { de la sistematización } \\
\text { de experiencias } \\
\text { cotidianas. }\end{array}$ & 0 & 10 & 70 & 20 & 100 \\
\hline $\begin{array}{l}\text { Contenidos } \\
\text { Teóricos }\end{array}$ & $\begin{array}{l}\text { El saber en el } \\
\text { manejo de las TIC } \\
\text { es el resultado de } \\
\text { la aplicación de } \\
\text { contenidos teóricos y } \\
\text { conceptuales. }\end{array}$ & 0 & 10 & 80 & 10 & 100 \\
\hline $\begin{array}{c}\text { Combinación } \\
\text { de } \\
\text { Experiencia y } \\
\text { Teoría }\end{array}$ & $\begin{array}{l}\text { La combinación de la } \\
\text { experiencia y la teoría } \\
\text { es los que me ha } \\
\text { permitido consolidar } \\
\text { mis saberes en el } \\
\text { manejo de las TIC }\end{array}$ & 0 & 20 & 60 & 20 & 100 \\
\hline
\end{tabular}

Fuente: Docente Investigadora. 2019

- $\quad$ El $70 \%$ de los docentes tienen un adecuado manejo de las TIC, gracias a la sistematización de sus experiencias pedagógicas cotidianas, que inciden en la apropiación de dicho conocimiento en el uso de las nuevas tecnologías de la información y la comunicación.

- En referencia a la necesidad de recurrir a la aplicación de contenidos teóricos existente sobre TIC, un $80 \%$ lo han hecho para fundamentar su utilización y mejorar la práctica pedagógica innovadora.

- $\quad$ Por otra parte, un $60 \%$ han recurrido a la combinación de las dos estrategias Sistematización y aplicación de contenidos teóricos) para fundamentar ese conocimiento y saber especializado en manejo y uso de las TIC en el aula escolar. 


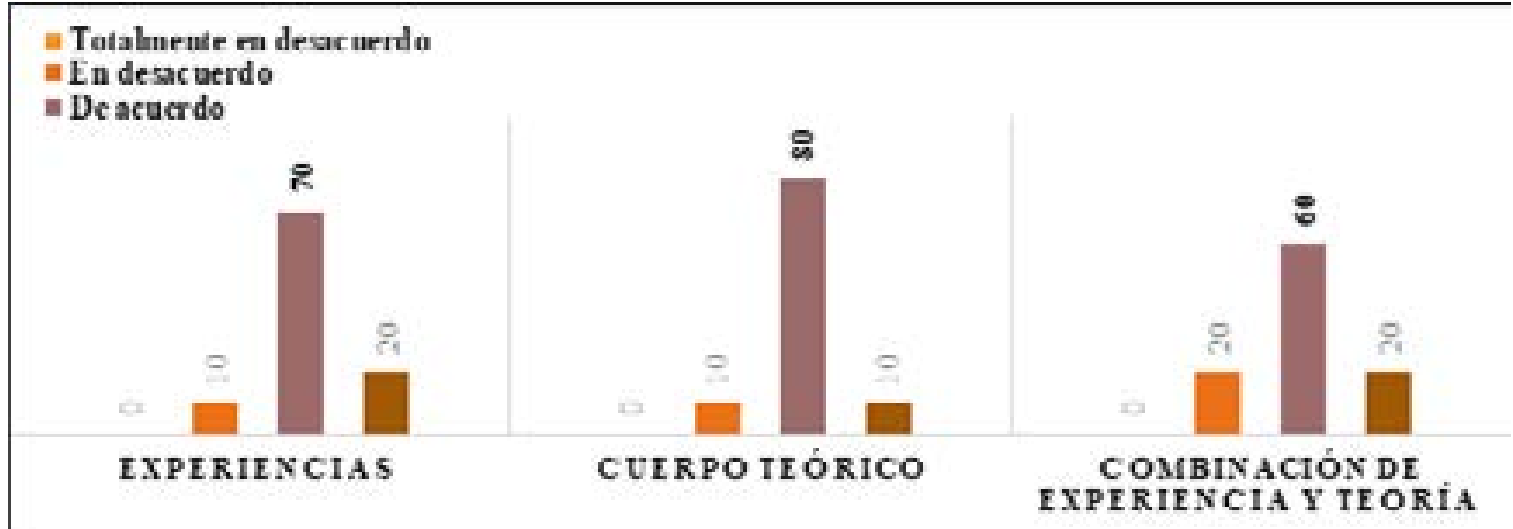

Gráfico 3. Porcentaje de aplicación del saber especializado por parte de docentes.

Fuente: Docente Investigadora. 2019

\subsubsection{Aporte del saber pedagógico al uso y manejo de las TIC y desarrollo del capital humano de la Institución Educativa La Chicueña, municipio de El Tambo Cauca}

A partir de los resultados obtenidos en esta categorización, los docentes de esta Institución Educativa, han accedido a un conocimiento en el manejo y uso de las TIC, más por iniciativa y necesidad propia, que por la existencia de una cultura hacia la innovación pedagógica tecnológica para el desarrollo de su ejercicio profesional. No obstante, el conocimiento adquirido ha fortalecido el ejercicio pedagógico y viene incidiendo en el crecimiento del capital humano de la Institución, inicialmente en el docente y posteriormente en los estudiantes y comunidad educativa, quienes perciben con mayor motivación y expectativa la clase dentro del aula.

Desde esta perspectiva, los docentes más conocedores de las TIC, ayudan a aquellos que aun presentan dificultades, logrando así el fortalecimiento del capital humano frente al manejo de las TIC, abordando aspectos básicos, resolviendo inquietudes y fortaleciendo habilidades de los participantes de los talleres, al igual sistematizando experiencias en las que además logra reforzar este saber mediante la aplicación de los contenidos teóricos y conceptuales, así como, por la combinación de estas experiencias con el conocimiento adquirido.

\subsection{Uso y Manejo de las TIC en la Gestión del Conocimiento}

Tratadistas, citados por De Aparicio (2009), determinan que el conocimiento constituye el activo más valioso de una organización en la actual la sociedad de la información, por cuanto, permite la conjunción de esta con la economía del conocimiento, que articulada al aporte que hace el proceso educativo de una comunidad, permiten un acelerado desarrollo de competencias con las que el ser humano puede realizar las transformaciones que la sociedad requiere. En tal sentido, indagados los docentes del Centro Educativo sobre la información que tienen respecto de la gestión del conocimiento y la importancia de este en el cometido escolar, así como en el conocimiento de las innovaciones tecnológicas, su uso y manejo en el ámbito de la práctica pedagógica en el contexto educativo rural, se encontró que: 
Tabla 4. Tabulación sobre gestión del conocimiento de los docentes del Centro Educativo

\begin{tabular}{|c|c|c|c|c|c|c|}
\hline Subcategoría & Pregunta & $\begin{array}{c}\text { Totalmente } \\
\text { en } \\
\text { Desacuerdo } \\
\%\end{array}$ & $\begin{array}{c}\text { En } \\
\text { Desacuerdo } \\
\%\end{array}$ & $\begin{array}{c}\text { De } \\
\text { Acuerdo \% }\end{array}$ & $\begin{array}{c}\text { Totalmente } \\
\text { de Acuerdo } \\
\%\end{array}$ & Total \\
\hline $\begin{array}{c}\text { Información } \\
\text { sobre el Tema }\end{array}$ & $\begin{array}{c}\text { Considero que tengo } \\
\text { información sobre lo } \\
\text { que es la gestión del } \\
\text { conocimiento en TIC }\end{array}$ & 0 & 60 & 30 & 10 & 100 \\
\hline La & $\begin{array}{l}\text { Considero que } \\
\text { la gestión del } \\
\text { conocimiento TIC es } \\
\text { un tema importante } \\
\text { en la gestión escolar }\end{array}$ & 0 & 10 & 50 & 40 & 100 \\
\hline
\end{tabular}

Fuente: Docente Investigadora. 2019

- Un 60\% no dispone de una conceptualización clara y referente sobre el termino gestión del conocimiento, lo cual, frente al uso de las TIC, es preocupante toda vez, que esta gestión es la que incide en el desarrollo de la comunidad educativa.

- $\quad$ No obstante, un $50 \%$ considera que la conceptualización es importante en la gestión escolar.

- Un $40 \%$ de los docentes plantean que en el contexto de la sociedad y de la escuela moderna, es imprescindible desarrollar habilidades y competencias respecto de aprovechamiento de las TIC en la escuela y en el contexto de la comunidad educativa.

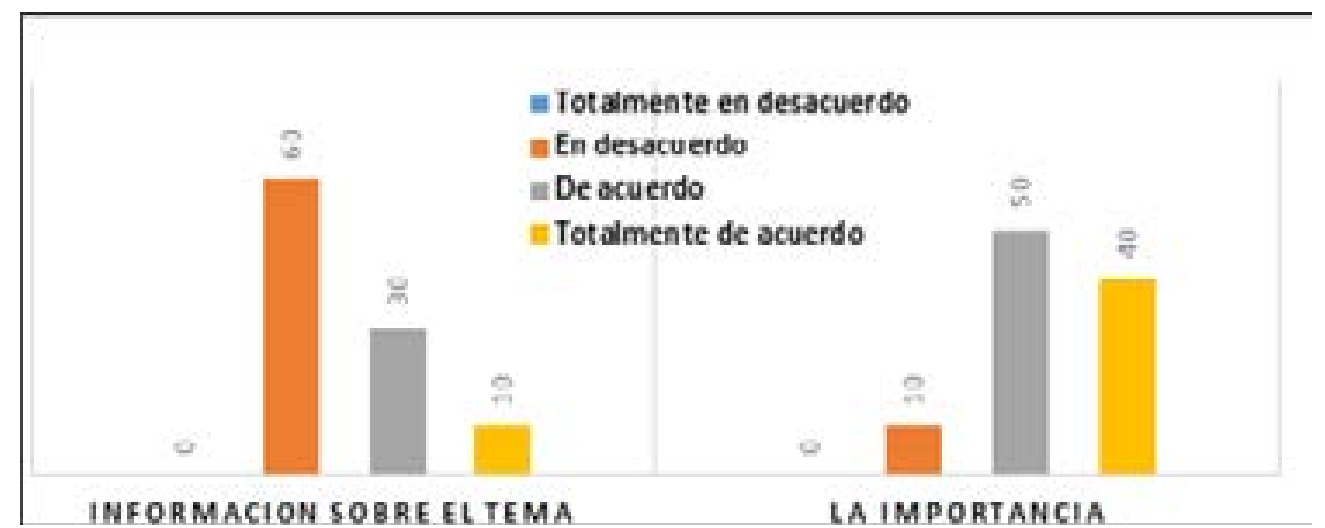

Gráfico 4. Porcentajes de apropiación conceptual de la gestión del conocimiento Fuente: Docente Investigadora. 2019 
4.2.1 Aporte de la gestión del conocimiento en TIC al desarrollo del capital humano y de la Institución Educativa La Chicueña, municipio de El Tambo Cauca.

El profesor de hoy es un gestor o facilitador de la gestión del conocimiento, en cuya gestión, el estudiante juega un papel importante, por cuanto, es también el constructor de su propio conocimiento. De lo anterior y, avocando la importancia del uso de la tecnología en la gestión de ese conocimiento, el docente debe ocuparse por actuar, aprender y desarrollar nuevos contendidos, nuevas formas y nuevos instrumentos pedagógicos para que haya innovación constante en los modos de aprender y de enseñar, promoviendo en la actual sociedad del conocimiento, una educación con creatividad, flexibilidad, motivación e iniciativa en el proceso educativo.

En esta medida, la gestión al aporte que hace el aprendizaje en el uso y manejo de las TIC por parte del docente actual, permite para él, no solo dotarse de un conocimiento adicional, sino de asociar nuevos aprendizajes, nuevas formas de orientación de clase, de planeación pedagógica Sánchez, et al (2018), preparación para la resolución de problemas, la puesta en marcha del trabajo colaborativo y ganar en confianza profesional de su desempeño, así como profundizar en el ejercicio investigativo del contexto en el que se desenvuelve.

\subsection{Capital Humano}

Considerando a Nonaka y Takeuchi (1995) en su texto "Teoría de la creación del conocimiento organizacional", la creación del conocimiento es un proceso dinámico, humano de justificación personal de creencias hacia la verdad, que se desarrolla a partir de dos dimensiones y que determina el nivel de conocimiento que adquiere el hombre. (p. 58)

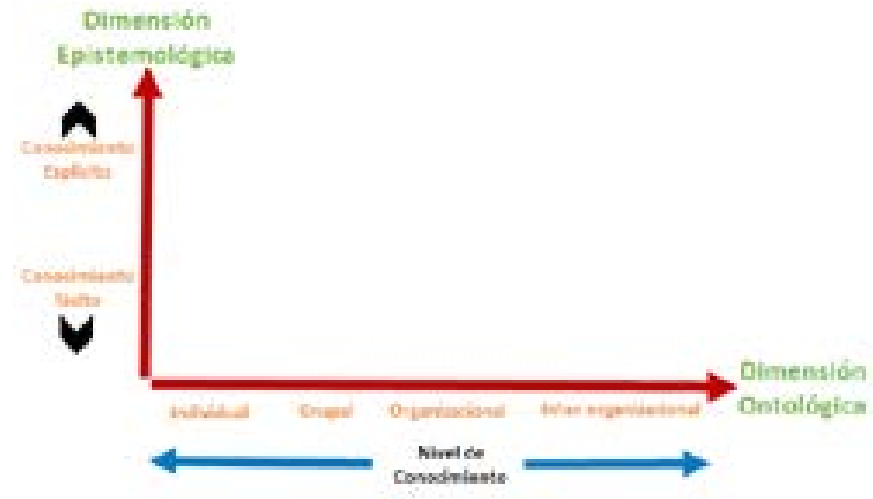

Ilustración 1. Dimensiones de la creación de conocimiento según Nonaka y Takeuchi Fuente: Docente Investigadora, a partir de http://anamariaaguilera.com/nonaka-ytakeuchi/. 2019

En virtud de lo anterior, en cualquier organización y en particular en las Instituciones Educativas, el capital humano es el gestor de conocimiento, a partir de la labor y acción de sus principales agentes, los docentes, quienes por deducción son generadores y gestores del conocimiento, ya sea individual, grupal, organizacional e inter organizacional que, contextualizado a la escuela, determinan unos aprendizajes en el docente.

Así mismo, los estudiantes en formación como sujetos del aprendizaje quienes se encuentran dedicados a aprender a aprender y a procesar el conocimiento que los docentes les trasmiten, de acuerdo con las estrategias pedagógicas particulares, son fundamentales en desarrollo del capital social de una comunidad en general, mediante la modificación de parámetros culturales, generadores del crecimiento y desarrollo de la comunidad educativa en general.

En tal sentido, indagados los docentes del Centro Educativo, sobre la capacidad que disponen como talento humano en conocimientos y habilidades asertivas de manejo y uso de las TIC como elemento dinamizador de la práctica docente dentro del aula, se encontró que: 
Tabla 5. Importancia del talento humano dentro del Centro Docente La Chicueña.

\begin{tabular}{|c|c|c|c|c|c|c|}
\hline Subcategoría & Pregunta & $\begin{array}{l}\text { Totalmente } \\
\text { en } \\
\text { Desacuerdo }\end{array}$ & $\begin{array}{c}\text { En } \\
\text { Desacuerdo }\end{array}$ & $\begin{array}{c}\text { De } \\
\text { Acuerdo }\end{array}$ & $\begin{array}{c}\text { Totalmente de } \\
\text { Acuerdo }\end{array}$ & Total \\
\hline $\begin{array}{c}\text { Información } \\
\text { sobre el } \\
\text { Tema }\end{array}$ & $\begin{array}{l}\text { Considero } \\
\text { que el capital } \\
\text { humano } \\
\text { actualmente } \\
\text { cuenta con } \\
\text { herramientas, } \\
\text { conocimientos } \\
\text { y habilidades } \\
\text { asertivas TIC } \\
\text { dentro de una } \\
\text { institución } \\
\text { educativa }\end{array}$ & 0 & 30 & 50 & 20 & 100 \\
\hline $\begin{array}{c}\text { La } \\
\text { Importancia }\end{array}$ & $\begin{array}{l}\text { Considero que } \\
\text { el papel que } \\
\text { desempeña } \\
\text { el capital } \\
\text { humano es } \\
\text { importante } \\
\text { en las } \\
\text { instituciones } \\
\text { educativas, a } \\
\text { partir del uso y } \\
\text { manejo de las } \\
\text { TIC. }\end{array}$ & 0 & 0 & 20 & 80 & 100 \\
\hline
\end{tabular}

Fuente: Docente Investigadora. 2019

- Un $50 \%$ de los docentes manifiestan contar con conocimientos sobre uso de TIC y la aplicación de herramientas pedagógicas dentro del aula de clase, que fortalecen el capital humano de la Institución y por consiguiente el desarrollo de su labor docente y formativa.

- $\quad$ El $30 \%$ aun no cuentan con elementos de información sobre el tema, lo cual, implica que no en todas las sedes del Centro Educativo, puedan estarse adelantando acciones pedagógicas usando estas nuevas tecnologías de la información y la comunicación.
- $\quad$ El 80\% consideran importante la gestión del talento humano de todo el Centro Educativo, orientado a la aplicación del uso y manejo de las TIC, no solo como herramienta pedagógica, sino como, instrumento de fundamentación del conocimiento de toda la comunidad educativa (Directivo Docente, Docente, Estudiante, Padre de Familia, Comunidad en Generall. Esto implica que, los docentes que hacen parte la Institución Educativa, si consideran importante que todo el capital humano de las sedes, conozca, se forme o capacite en la gestión TIC, para que dicho conocimiento, incida favorablemente en el desarrollo pedagógico de la formación del resto 
del talento humano que hace uso de la escuela, así como de su entorno social y cultural.

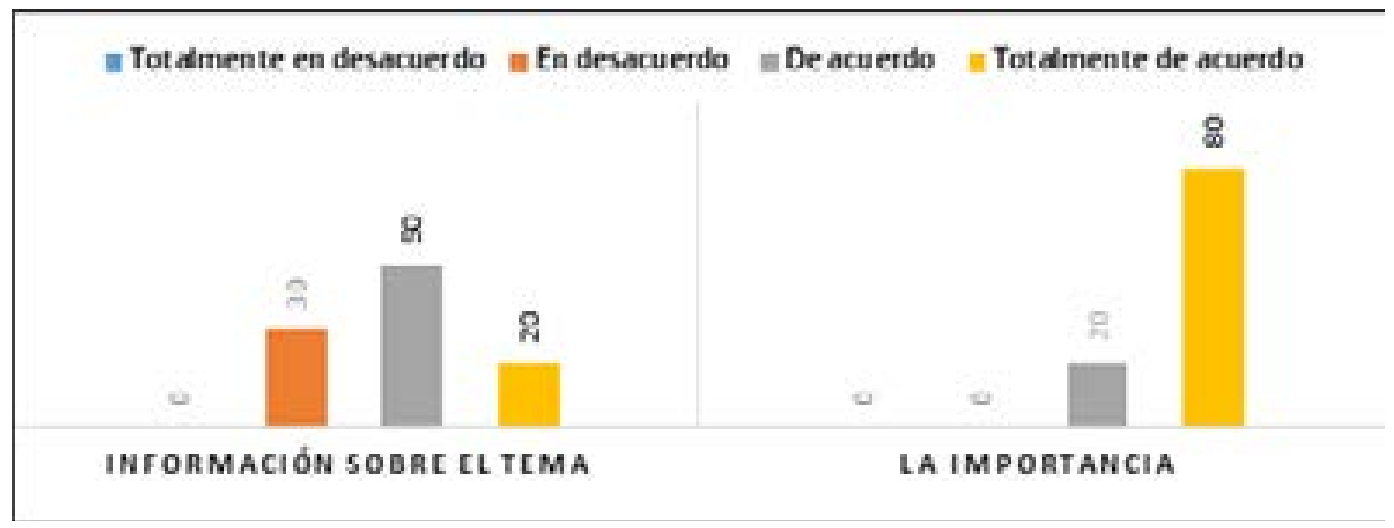

Gráfico 5. Porcentaje de apropiación del TIC por parte del Talento Humano del Centro Educativo La Chicueña

Fuente: Docente Investigadora. 2019

\subsubsection{Promoción de la gestión del capital humano frente al manejo de las TIC.}

Un cuerpo docente formado o capacitado en el uso y manejo de las TIC, puede favorecer el desarrollo pedagógico y estructural de toda la comunidad educativa del Centro Docente, así como el fortalecimiento del rol de docente en el aula de clase. En este sentido y buscando contribuir al propósito de la promoción de la gestión del capital humano frente a las TIC, surge desde la investigadora, la iniciativa de adelantar un ejercicio de capacitación, mediante el desarrollo de cuatro talleres prácticos con docentes frente al manejo y uso de las TIC en el aula de clase, considerando que debido a la distancia del Centro Educativo a la cabecera municipal o la ciudad de Popayán, amerita realizarlo en la sede principal de la Institución Educativa.

\section{DISCUSIÓN}

Existe la necesidad de implementar acciones estratégicas que permitan al docente de hoy y más al que trabaja en el contexto rural de los municipios, adelantar formación consecuente con el uso y manejo de estas tecnologías actuales para que puedan servir al mejoramiento no solo del talento humano del Centro o Institución Educativa, sino del proceso de enseñanzaaprendizaje y por consiguiente al desarrollo de toda la comunidad y de la región donde cohabita la escuela.

El desarrollo de las nuevas tecnologías de la información y la comunicación (TIC) cada vez ameritan nuevos retos y oportunidades en el ámbito del proceso educativo y que la institución escolar, está enfrentando la incorporación de dichas tecnologías en el campo de la enseñanza y en la práctica pedagógica de distintas maneras y a partir de diferentes estrategias y programas sean estatales o institucionales.

Sin embargo, preocupa que, en la implementación de estas TIC, se debe disponer de factores y elementos mínimos en la escuela, como son: la gestión del conocimiento, el desarrollo del saber del docente en esta área del conocimiento, los equipos e instrumentos que permitan la interacción de dicho saber con las nuevas tecnologías, y además la verificación permanente para determinar si esta implementación se está llevando correctamente o si por el contrario, existen en la comunidad educativa, factores - circunstancias que impiden el desarrollo 
o utilización de las nuevas tecnologías de la información o la comunicación.

Existen en la gestión TIC en la escuela, fracturas operativas, al no disponer de los elementos o equipos básicos como la conectividad a internet de manera permanente y con buena velocidad, computadores, video beam, entre otros y del conocimiento por cuanto, algunos docentes no cuentan con la formación formal o informal al respecto del uso y manejo de las TIC.

Si bien, la práctica pedagógica del aula, requiere de la innovación mediante el uso de las TIC que la haga más agradable e interactiva en la relación docente - estudiantes, se debe considerar que el proceso educativo se enfoque no solo en el uso de estas tecnologías, sino también que la actual sociedad de la información que requiere de docentes con otras habilidades y un proceso continuo de formación, se preparen para el uso y apropiación de las TIC, logrando así una transformación de conocimiento.

Así mismo, las instituciones educativas no se transforman por si solas, sino que es transformación es gracias al capital humano que poseen y que si a ellas y a ello, se les incorporan instrumentos tecnológicos y elementos didácticos que hagan válido el uso de las TIC, escuela como institución social de formación y educación, sin lugar a duda cambiará.

Por ello, corresponde a las instituciones gubernamentales (Nacionales, departamentales y locales), de la gestión administrativa del Directivo Docente y de toda la comunidad educativa del Centro Docente, propender por la dotación de los medios físicos y tecnológicos necesarios para la implementación del uso de las TIC en la escuela rural.

En tanto que es responsabilidad y tarea de los docentes del Centro Educativo, continuar con su formación permanente a la optimización de dichos recursos, tanto en la planificación de la práctica pedagógica, así como en los contenidos y los objetivos educativos y de proyección social de la comunidad educativa. Así como, realizar una adecuada gestión de las TIC que puedan disponerse en el Centro Docente, para evitar lo que se ha observado en otras Instituciones Educativas, donde a pesar de disponer de los equipos y elementos tecnológicos, estos están siendo subutilizados y almacenados a merced del deterioro y la obsolescencia.

Es fundamental dentro del ejercicio del manejo y uso de las TIC, determinar como elemento de valoración, la divulgación y promoción de aquellas experiencias docentes exitosas que puedan desarrollarse en las Instituciones Educativas, con el fin de, no solo, valorar el impacto de la utilización de diversas estrategias pedagógicas, sino establecer los aspectos más relevantes que contribuyan al mejoramiento de su propia experiencia.

\section{REFERENCIAS BIBLIOGRÁFICAS}

Achilli, E. (1986). La práctica docente: una interpretación desde los saberes del maestro, Cuadernos de Formación Docente, Universidad Nacional de Rosario. Bogotá. 16 p.

Barrón, C. (2006). Los saberes del docente. Una perspectiva desde las humanidades y las ciencias sociales. Perspectiva educacional, formación de profesores. Pontificia Universidad Católica de Valparaíso. Chile.

Danieli, M. (2018) Enseñar Tecnología con TIC: saberes docentes y trayectorias. Recuperado el 12 de noviembre de 2018, en Medina Rincón, I. L. Saberes de los docentes de dos instituciones educativas públicas del departamento de Boyacá ante las dificultades de aprendizaje que 
afectan el desempeño académico de sus estudiantes (Doctoral dissertation, Universidad Nacional de ColombiaSede Bogotá).

De Aparicio, X. (2009). La gestión del conocimiento y las tics en el siglo XXI. CONHISREMI, Revista Universitaria de Investigación y Diálogo Académico, Vol. 5, No. 1. Universidad Nacional Experimental. Miranda. Venezuela.

Delgado, J. (2012). Implicaciones presentadas por el uso de alternativas tecnológicas y organizativas para un desarrollo autogestionario del sujeto educable en contextos de ruralidad (Doctoral dissertation). Universidad Católica de Manizales.

Díaz, L. (2007). Gestión del conocimiento y del capital intelectual: una forma de migrar hacia empresas innovadoras, productivas y competitivas. Revista Escuela de Administración de Negocios, núm. 61, septiembre-diciembre, pp. 3967 Recuperado en: http://www.redalyc. org/pdf/206/20611495005.pdf

Guerrero, M. (2014). Metodologías activas y aprendizaje por descubrimiento. Las TIC y la educación. Marpadal Interactive Media S.L.

Hernández, R. (2010). Metodología de la investigación sexta edición edit. Macgraw Hill. México.

Jara, S. y Dörr, A. (sf) Procesos afectivos. Extraído de https://tuvntana.files.wordpress. com/2015/06/apunte-procesos_ afectivos.pdf
Linares, M. (2013) Capital humano, gestión académica y desarrollo organizacional. Educ Med Super, Set 2013, vol.27, no.3, p.288-295. ISSN 0864-2141. Recuperado en: http://www.ems.sld.cu/index.php/ ems $\% 20 / a r t i c l e / v i e w / 231 / 117$

Mendoza, J. (2002), “Educación, experiencia y especialización manufacturera en la frontera norte de México", en Revista Comercio Exterior, vol. 52, núm. 4, abril, México.

Ministerio de Educación Nacional. (sf)Ser maestro hoy: El sentido de educar y el oficio del maestro. Revolución educativa: Al tablero._http://www.mineducacion. gov.co/1621/propertyvalues-31232_ tablero_pdf.pdf

Muñoz. P. y Muñoz, I. (2001). Intervención de la familia. Estudios de casos. En Pérez Serrano, G. (coord.) Modelos de investigación cualitativa en educación social y animación sociocultural: aplicaciones prácticas. Madrid: Narcea.

Nonaka, I. y Takeuchi, H. (1997). The knowledgecreating company. Oxford University Press, Nueva York.

Porlán, R. \& Martín, R. (1999). Tendencias en la formación inicial del profesorado. Universidad Complutense de Madrid - Universidad de Sevilla. Revista Interuniversitaria.

Pozo, J. \& Monereo, C. (1999). Un currículo para aprender. Profesores, alumnos y contenidos ante el aprendizaje estratégico. Madrid: Santillana: Aula XXI.

Quecedo, R. y Castaño, C. (2002) Introducción a la metodología de investigación cualitativa Revista de Psicodidáctica, núm. 14, 2002, 
pp. 5-39 Universidad del País Vasco/ Euskal Herriko Unibertsitatea VitoriaGazteis, España. Recuperado el 09 de noviembre de 2018, en: http://www. unilibrebaq.edu.co/unilibrebaq/images/ CEUL/mod3recoleccioninform.pdf.

Sánchez, G.D.C.;_Diosa, G.J.; Ávila, G.L.M; Hernández, R. M del P. (2018). La planeación pedagógica para la atención integral en la primera infancia. Revista Texztos y sentidos No. 17. (p.155-177).

Sánchez-Amaya, T. y González-Melo, H. S. (2016). Saber pedagógico: fundamento del ejercicio docente. Educ. Educ., 79(2), 241-253. DOI:10.5294/edu.2016.19.24

Strauss, A. y Corbin, J. (1990). Basics of qualitative research: Grounded theory procedures and techniques, London: Sage.

Tique, E. (2011). Estado actual de la aplicación de las TIC en los colegios que imparten el ciclo de educación media de las zonas rurales del municipio de Ibagué. Universidad del Tolima

Vesga-Parra, D. \& Hurtado-Herrera, D. (2013). La brecha digital: representaciones sociales de docentes en una escuela marginal. Revista Latinoamericana de Ciencias Sociales, Niñez y Juventud, 11(1), 137-149.

Velasco, S. (2012). Análisis comparativo de las políticas públicas de innovación en Colombia 1968-2012. Universidad Militar Nueva Granada. 